OPEN ACCESS

Edited by:

Tobias Hayer,

University of Bremen, Germany

Reviewed by:

Henry W. Chase

University of Pittsburgh, USA

Diane Carol Gooding

University of Wisconsin-Madison, USA

Susana Jiménez-Murcia,

Bellvitge University Hospital, Spain

*Correspondence:

Giovanna Nigro

giovanna.nigro@unicampania.it

Specialty section:

This article was submitted to

Psychopathology,

a section of the journal

Frontiers in Psychology

Received: 12 January 2017 Accepted: 15 March 2017

Published: 03 April 2017

Citation:

Nigro G, Cosenza M and Ciccarelli $M$

(2017) The Blurred Future of

Adolescent Gamblers: Impulsivity,

Time Horizon, and Emotional Distress.

Front. Psychol. 8:486.

doi: 10.3389/fpsyg.2017.00486

\section{The Blurred Future of Adolescent Gamblers: Impulsivity, Time Horizon, and Emotional Distress}

\author{
Giovanna Nigro *, Marina Cosenza and Maria Ciccarelli \\ Department of Psychology, Università degli Studi della Campania Luigi Vanvitelli, Caserta, Italy
}

The main purpose of this study was to investigate the interplay of functional and dysfunctional impulsivity, delay discounting, time perspective, and emotional negative states on gambling severity in Italian adolescents. A second aim of the study was to analyze the developmental trajectories of gambling involvement, functional and dysfunctional impulsivity, delay discounting, consideration of future consequences, and negative affectivity in a cross-sectional perspective. One thousand and ten Italian adolescents aging between 12 and 19 years were administered the South Oaks Gambling Screen Revised for Adolescents (SOGS-RA), the Functional and Dysfunctional Impulsivity Scale (FDIS), the Monetary Choice Questionnaire (MCQ), the Consideration of Future Consequences Scale (CFC-14), and the Depression, Anxiety and Stress Scales-21 (DASS-21). Data analyses were conducted using correlational analysis, Chi-square test, analysis of variance, and hierarchical regression analysis. Results indicated that, relative to non-gamblers and non-problem gamblers, at-risk and problem gamblers showed higher levels of impulsivity, steeper delay discounting, shorter time horizon, and reported experiencing significantly higher levels of depression, anxiety, and stress. Results of hierarchical regression analysis, with SOGS-RA scores as the dependent variable, and gender, age, FDIS, MCQ, CFC-14, and DASS-21 scores as independent variables, indicated that, along with gender and age, low scores of future orientation and high scores of dysfunctional impulsivity, depression, anxiety, present orientation, and delay discounting significantly predicted gambling severity. These findings provide further evidence that the higher the gambling involvement, the greater the tendency to devalue delayed rewards and to focus on the immediate consequences of one's behavior. Interestingly, for the first time these results reveal an association between gambling severity and both dysfunctional impulsivity and negative affective states across adolescence. Finally, results of cross-sectional analyses suggest that gambling severity contributes more than age in shaping the developmental trajectories of functional and dysfunctional impulsivity, delay discounting, time perspective, and negative affective states.

Keywords: gambling, adolescence, impulsivity, delay discounting, temporal perspective, depression, anxiety, negative affect 


\section{INTRODUCTION}

In the last decades gambling addiction has become a serious public health issue. Mainly due to the increasing availability of online gambling and the similarity between modern forms of gambling and other familiar technology-based games, the prevalence of disordered gambling will predictably increase further in the near future (Donati et al., 2013; McCormack et al., 2014; Delfabbro et al., 2016). Recently, Gainsbury et al. (2016b) have demonstrated that for a large proportion of at-risk and problem gamblers the exposure and the engagement with social media advertisements for gambling worsened their problems (see also Gainsbury et al., 2016a).

In this backdrop, adolescent participation in gambling activities is of particular concern, given that some risk factors for disordered gambling are so manifest during adolescence, that adolescence by itself may be regarded as a risk factor for the onset and the development of problematic gambling (Messerlian et al., 2004, 2005; van den Bos et al., 2013).

Indeed, large-scale international prevalence surveys and metaanalytic studies have shown that $10-15 \%$ of adolescents are at risk for developing gambling problems and 3-8\% can be considered having serious gambling problems (see Blinn-Pike et al., 2010; Ladouceur et al., 2013). A recent review on the prevalence of adolescent problem gambling across five continents reported that $0.2-12.3 \%$ of youth meet diagnostic criteria for problem gambling (Calado et al., 2016). In spite of gambling is an illegal activity in Italy under the age of 18, some studies on Italian adolescents have found that $16-17 \%$ of high school students were at-risk of developing problem gambling, and $7-8 \%$ problem gamblers (Chiesi et al., 2013; Cosenza et al., 2014; Cosenza and Nigro, 2015).

Even if studies on adolescent gambling increased in the last years, research on risk factors from early to late adolescent gambling remains very scarce. This is even more surprising since several studies have highlighted that adolescents represent a high vulnerable population and research has demonstrated that, other things being equal, severe gambling-related difficulties in adulthood steam from early gambling problems (Blinn-Pike et al., 2010; Volberg et al., 2010; Olason et al., 2011; Cosenza et al., 2014; Gupta and Derevensky, 2014).

While there are several potential factors leading to the onset and development of problematic gambling, the research on the identification of risk factors associated with youth disordered gambling is still limited in quantity (Shead et al., 2010; ScholesBalog et al., 2014). Furthermore, to date the interplay of different risk factors on adolescent problem gambling has not been adequately taken into account (Cosenza and Nigro, 2015).

Although the etiology of gambling disorder is complex and multifaceted, several studies have identified impulsivity as the most robust characteristic associated with disordered gambling (MacKillop et al., 2014). Interestingly, prospective investigations have indicated that high impulsivity during early adolescence predicts later gambling problems (Pagani et al., 2010; Shenassa et al., 2012; Slutske et al., 2012).

Impulsivity describes a constellation of heterogeneous traits or behavioral dispositions that includes inability to take into account the future consequences of current behavior and the tendency to devalue delayed rewards. Acting without considering future consequences has been considered one of the potential determinants of impulsive behavior (Whiteside et al., 2005; see also Sharma et al., 2013). Likewise, delay discounting, that is the relative preference for small immediate rewards, has been considered a behavioral index of impulsivity (Ainslie, 1975; Madden et al., 2009; see also Amlung and MacKillop, 2011; Gray and MacKillop, 2014). Studies examining the relation between gambling and delay discounting among late adolescents indicated that, relative to non-problem gamblers, young problem gamblers more rapidly discounted delayed monetary outcomes (for exception see Holt et al., 2003; MacKillop et al., 2006; Cosenza and Nigro, 2015; Nigro and Cosenza, 2016).

The association between pathological gambling and shortened time horizon was first investigated by Hodgins and Engel (2002). Subsequent studies further supported the existence of a positive association between disordered gambling and insensitivity to future consequences among both adult (Toplak et al., 2007; MacLaren et al., 2012; Ciccarelli et al., 2016b) and adolescent gamblers (however, for different results, see MacKillop et al., 2006; Cosenza and Nigro, 2015; Cosenza et al., 2016).

Finally, as indicated by earlier studies, negative emotional states, such as depression, anxiety, and stress, are significant correlates of problematic gambling (Blaszczynski and McConaghy, 1989; Coman et al., 1997; Blaszczynski and Nower, 2002; El-Guebaly et al., 2006; Kim et al., 2006; Ladouceur et al., 2006; Johansson et al., 2009; Barrault and Varescon, 2013; Lorains et al., 2014; Dowling et al., 2015; Raylu et al., 2016; Toneatto and Pillai, 2016). In particular, some epidemiological studies indicated that problematic gambling is often associated with mood disorders (Griffiths, 1995; see also Lorains et al., 2011), as well as that pathological gamblers in treatment frequently suffer from clinical depression (i. e., Ladouceur et al., 2006). Nower and Blaszczynski (2010) hypothesized that gambling contributes to alleviate negative emotional states or boredom (Wulfert et al., 2005; Wood and Griffiths, 2007; see also Stewart et al., 2008), whereas Gee et al. (2005) observed that gambling increases anxiety.

From the few studies investigating the co-occurrence of negative affects and gambling in adolescence emerged that, relative to both non-gamblers and social gamblers, adolescent problem gamblers have higher rates of depression, females have significantly higher rates of depression than males, and older adolescents score higher than younger (Nower et al., 2004). Furthermore, compared to non-gamblers, social and atrisk gamblers, adolescent problematic gamblers report higher level of both state and trait anxiety and social stress, with females obtaining higher scores than males (Ste-Marie et al., 2006). In a sample of young online gamblers Matthews et al. (2009) found that problem gambling was significantly predicted not only by negative mood states after gambling, but also by negative mood states in general. More recently, in a longitudinal study involving adolescents and early adults, Dussault et al. (2011) demonstrated that the association between depression and problematic gambling in adolescence steams mainly from impulsivity. In addition, the mechanisms explaining 
the association between the two disorders vary as a function of developmental stages.

Although evidences from previous research support the idea that there could be a complex interplay among problematic gambling, impulsivity, "myopia for the future," and negative emotional states in adolescence, to date no study has ever examined the interrelationship among these variables all together.

The main aim of the present study was to investigate the interplay among impulsivity, delay discounting, time perspective, and negative affectivity in a large sample of adolescents aging between 12 and 19 years. A second aim of the present study was to analyze the developmental trajectories of gambling involvement, functional and dysfunctional impulsivity, delay discounting, consideration of future consequences, and negative affectivity in a cross-sectional perspective.

In line with previous research on both adults and adolescents, it was expected that female adolescents would be less likely to report gambling-related problems than male adolescents. Moreover, it was hypothesized that the more severe the gambling involvement is, the higher the level of impulsivity, the steeper the delay discounting rates, and the shorter the time horizon are. Finally, it was also hypothesized that, relative to other groups, atrisk and problem gamblers would show more severe depression, anxiety, and stress symptoms.

\section{METHODS}

\section{Participants}

One thousand and ten Italian students (47,5\% males) aged between 12 and 19 years (Mean age $=15.37$ years; $S D=$ $2.05)$ attending public middle $(14.2 \%)$ or high school $(58.4 \%$ lyceum and $27.4 \%$ technical and trade school) in Southern Italy took part in the study. They were administered the South Oaks Gambling Screen Revised for Adolescents (Winters et al., 1993, 1995; Italian version: Colasante et al., 2013; SOGSRA), the Functional and Dysfunctional Impulsivity Scale (FDIS; Dickman, 1990), the Monetary Choice Questionnaire (Kirby and Marakovic, 1996; Kirby et al., 1999; MCQ), the Consideration of Future Consequences Scale (Joireman et al., 2012; Italian validation: Nigro et al., 2016; CFC-14), and the Depression, Anxiety and Stress Scales-21 (Lovibond and Lovibond, 1995; Italian validation: Bottesi et al., 2015; DASS-21). Participants did not receive anything for participating in the study. The authors administered the questionnaires. For each measure participants received detailed written instructions. Participants were allowed to ask any questions about the questionnaires, if any.

\section{Measures}

Adolescent gambling behavior was measured through the SOGS-RA, the most widespread self- report instrument for assessing the prevalence of problem gambling in adolescence. The questionnaire is made up of 12 scored items measuring gambling behavior and gambling-related problems during the past 12 months. The total score ranges from a minimum of 0 to a maximum of 12 . The un-scored SOGS-RA items request participants to indicate, among others, the frequency of participation in different gambling activities, the largest amount of money gambled in 1 day, and parental involvement in gambling. In addition, we asked participants to specify the primary motives for gambling from a list (Volberg, 1993). The Italian version of the SOGS-RA was found to have acceptable internal reliability ( $\alpha=0.78$; Colasante et al., 2013).

The FDIS is a 23 items self-report questionnaire assessing functional and dysfunctional impulsivity. The Functional Impulsivity scale (FI) consists of 11 items measuring the tendency to act quickly without planning when the situation demands it for personal gain. The Dysfunctional Impulsivity scale (DI) consists of 12 items assessing the tendency to engage in rapid, error-prone information processing in situations where slower methodical approaches are required. Respondents are asked to indicate the extent to which they agree with each statement on a 5-point scale ranging from 1 (strongly disagree) to 5 (strongly agree). In the present study, Cronbach's alpha for the functional and dysfunctional scales was 0.71 and 0.76 , respectively.

The MCQ is a measure of delayed reward discounting that presents participants with 27 hypothetical choices between a smaller reward available immediately, and a larger reward available at some point in the future, with delays ranging from 7 to 186 days. The 27 items are grouped into three categories on the basis of the approximate magnitudes of the delayed rewards. The three levels of magnitude are: small (\$25-\$35), medium (\$50-\$60), and large (\$75-\$85). Participants are instructed to respond in the same manner as they would with real money. The pattern of responding can be used to determine an estimate of the participant's overall discounting rate parameter $(k)$, as well as temporal discounting of rewards at the three different levels of magnitude ( $k$ small, $k$ medium, and $k$ large). The higher the $k$-values, the greater the proportion of choices for the smaller immediate monetary rewards. Calculating separate discount rates for each level of magnitude allows estimating the magnitude effect on discount rates, i. e., the tendency for discount rates to decrease as a function of reward level (Green et al., 1981).

The CFC-14 is a 14-item scale that was developed to measure individual differences in the extent to which people evaluate the immediate as opposed to distant implications of current behaviors and events. Responses are made with a 7-point Likert scale ranging from 1 (extremely uncharacteristic of $m e$ ) to 7 (extremely characteristic of $m e$ ). The CFC-14 is a two factors scale with two dimensions, one assessing consideration of immediate consequences (CFC-I), the other tapping consideration of future consequences (CFC-F). The Cronbach's alphas for the Immediate and Future scales were 0.84 and 0.83 , respectively, in a large sample of Italian adolescents (Nigro et al., 2016).

The DASS-21 is a self-report measure assessing three related negative affective states, namely depression, anxiety, and stress. The Depression scale comprises items that assess symptoms characteristically associated with dysphoric mood, such as sadness, worthlessness, lack of interest or involvement, and low self-esteem. The Anxiety scale taps signs of physical arousal, symptoms of panic attacks, as well as subjective experience of fear. The Stress scale assesses symptoms, such as difficulty relaxing, impatience, and being easily upset, irritable, or 
overreactive. Respondents are asked to indicate how much each statement applied to them during the previous week on a 4-point Likert scale ranging from 0 to 3 . Higher scores indicate severe emotional distress. Cronbach's alphas were, respectively, 0.82 for the depression subscale, 0.74 for the anxiety dimension, 0.85 for the stress subscale, and 0.90 for the full scale (Bottesi et al., 2015).

\section{Procedure}

The study was approved by the Ethics Committee of the Department of Psychology of the Second University of Naples. Prior to participation, all participants gave written informed consent. For minors, informed consent was obtained from parents. Participants were tested in groups of 10 to 20 at a time in a quiet room in school. Administration of all instruments required from 20 to $30 \mathrm{~min}$.

\section{Statistical Analyses}

Data were analyzed with the IBM Statistical Package for the Social Sciences, version 20.0. The alpha significance level was set at $p<0.05$. All variables were initially screened for missing data, distribution abnormalities, and outliers (Tabachnick and Fidell, 2013). Minor missing data ( $<2 \%)$ for all variables were replaced with means. Responses from the MCQ were analyzed using the approach described by Kirby et al. (1999). Because the $k$-values were positively skewed, a natural log transformation was conducted and used for all analyses. Furthermore, given that the distribution of the SOGS-RA was positively skewed, square root transformation was performed on this variable so that assumptions of normality, linearity and homoscedasticity had been adequately met.

Pearson correlation co-efficients and partial correlations were calculated to examine the relationships among the study variables. For categorical data differences in percentages were compared with the Chi-square test. Univariate and mixed-model ANOVAs were used to assess mean differences on continuous variables. Post hoc single comparisons were performed using twotailed $t$-tests for dependent groups with Bonferroni correction for multiple comparisons $(p<0.05)$. The magnitude effect on the discounting task was examined using paired samples $t$-test. Finally, to reveal potential predictors of gambling behavior and gambling-related problems, we performed a hierarchical regression analysis with SOGS-RA scores as the dependent variable, and gender, age, FDIS, MCQ, CFC-14, and DASS-21 scores as independent variables. In order to control for the presence of multicollinearity, before interpreting the regression coefficients, we calculated the variance inflation factors (VIF), which were below the recommended cutoff of 10 ( $\max . \mathrm{VIF}=$ 1.876; Ryan, 1997).

\section{RESULTS}

The associations among variables were assessed first using Pearson's correlation coefficients. Subsequently, we tested for gender differences through univariate analyses of variance (ANOVAs). Results showed significant gender differences on the SOGS-RA, the FDIS Functional Impulsivity dimension, the three discounting rates of the MCQ, the CFC-14 Immediate subscale, with males outperforming females, and on the three dimensions of the DASS-21, with females scoring higher than males. Since age was positively correlated with SOGS-RA, MCQ, and DASS21 scores, to ascertain whether the measures correlated even after controlling for gender and age, partial correlations among the measures were calculated (see Table 1).

As Table 1 shows, correlations between SOGS-RA, FDIS, MCQ, CFC-14, and DASS-21 scores were moderate to strong in strength.

In accordance with Winters et al.'s original SOGS-RA scoring system $(1993,1995)$, respondents were classified in the following four categories: non-gamblers, that includes individuals who

TABLE 1 | Partial correlations among all variables controlling for gender and age.

\begin{tabular}{|c|c|c|c|c|c|c|c|c|c|c|}
\hline & 2 & 3 & 4 & 5 & 6 & 7 & 8 & 9 & 10 & 11 \\
\hline 1. SOGS-RA & $0.079^{\star}$ & $0.341^{\star *}$ & $0.111^{\star \star}$ & $0.108^{\star \star}$ & $0.136^{\star \star}$ & $0.243^{\star \star}$ & $-0.207^{\star \star}$ & $0.279^{\star \star}$ & $0.273^{\star \star}$ & $0.271^{\star *}$ \\
\hline \multicolumn{11}{|l|}{ FDIS } \\
\hline 2. Functional Impulsivity & & $0.279^{\star \star}$ & 0.038 & -0.012 & 0.012 & $0.157^{\star \star}$ & -0.056 & -0.047 & -0.038 & 0.037 \\
\hline 3. Dysfunctional Impulsivity & & - & 0.030 & 0.054 & 0.059 & $0.366^{\star \star}$ & $-0.224^{\star \star}$ & $0.253^{\star \star}$ & $0.229^{\star \star}$ & $0.275^{\star \star}$ \\
\hline \multicolumn{11}{|l|}{ MCQ } \\
\hline 4. $k$ small & & & - & $0.592^{\star \star}$ & $0.526^{\star \star}$ & $0.110^{\star \star}$ & -0.062 & -0.014 & 0.031 & 0.047 \\
\hline 5. $k$ medium & & & & - & $0.645^{\star \star}$ & $0.074^{\star}$ & -0.057 & 0.020 & $0.069^{\star}$ & 0.059 \\
\hline 6. $k$ large & & & & & - & $0.069^{\star}$ & $-0.097^{\star \star}$ & 0.049 & $0.077^{\star}$ & $0.064^{\star}$ \\
\hline \multicolumn{11}{|l|}{ CFC-14 } \\
\hline 7. Immediate & & & & & & - & 0.031 & $0.185^{\star \star}$ & $0.186^{\star \star}$ & $0.203^{\star \star}$ \\
\hline 8. Future & & & & & & & - & 0.019 & 0.013 & 0.039 \\
\hline \multicolumn{11}{|l|}{ DASS-21 } \\
\hline 9. Depression & & & & & & & & - & $0.666^{\star \star}$ & $0.713^{\star \star}$ \\
\hline 10. Anxiety & & & & & & & & & - & $0.673^{\star \star}$ \\
\hline 11. Stress & & & & & & & & & & - \\
\hline
\end{tabular}

${ }^{*} p<0.05 ;{ }^{* \star} p<0.01$. 
reported no past year gambling, non-problem gamblers (score of 0-1), at-risk gamblers (score between 2 and 3), and problem gamblers (score of 4 or more). Of the total sample, $21.6 \%$ were screened as non-gamblers, $51.5 \%$ as non-problem gamblers, $19 \%$ as at-risk gamblers, and $7.9 \%$ as problem gamblers. The percentages of common gambling activities as a function of the relative frequency of participation in each activity during the last twelve months are reported in Table 2. As regards the amount of money invested in a single episode of play results indicated that $15.3 \%$ of at-risk and problem gamblers spent 1 Euro or less, $62.6 \%$ between 1 and 10 Euros, $15.7 \%$ between 10 and 50 Euros, $3.8 \%$ between 50 and 100 Euros, and 2.7\% more than 100 Euros.

In order to determine whether gambling activities varied as a function of gender and age, after collapsing gambling activities in three main categories, namely "offline games only" (74\% of participants), "online games only" (1\% of participants), "both offline and online games" (25\% of participants), data were submitted to Chi-square analyses. Non-gamblers and 23 participants who did not specify the gambling activities in which they engaged were excluded from analyses. Chi-square test revealed no significant differences due to gender $\left(\chi^{2}(2, N=\right.$ $769)=5.87 ; p=.053)$, nor to age $\left(\chi^{2}(14, N=769)=10.51\right.$; $p=0.724)$.

Chi-square test was also used to ascertain whether there was an association between severity of gambling involvement and each motive for gambling. Obviously, participants who reported no past year gambling (non-gamblers) were excluded from analysis. Results indicated that at-risk and problem gamblers gamble significantly more to win money $\left(\chi^{2}(2, N=792)=\right.$ 27.99; $p<0.001)$, for excitement or as a challenge $\left(\chi^{2}(2, N=\right.$ $792)=17.39 ; p<0.001)$, to socialize $\left(\chi^{2}(2, N=792)=13.64\right.$; $p<0.01)$, and for fun or entertainment $\left(\chi^{2}(2, N=792)=8.45\right.$; $p<0.05)$.

Group differences on the FDIS, the MCQ, the CFC-14 scales, and on the DASS-21 scores were tested using mixed model ANOVAs. Gender and age were included as covariates in the analyses. Results of $4 \times 2$ repeated measures ANOVA, with SOGS-RA group as a between-subjects factor and scores on the two FDIS scales, yielded a significant main effect of SOGS-RA group $\left[F_{(3,1004)}=32.12 ; p<0.001 ; \eta_{\mathrm{p}}^{2}=0.088\right]$. Furthermore,

TABLE 2 | Percentages of common gambling activities as a function of frequency (12-months-prevalence).

\begin{tabular}{lccrrr}
\hline & Never & Less than monthly & Monthly & Weekly & Daily \\
\hline Cards & 27.05 & 63.99 & 20.14 & 10.52 & 5.35 \\
Horse or dog races & 89.60 & 4.16 & 2.34 & 2.73 & 1.17 \\
Sports betting & 43.56 & 17.95 & 9.23 & 23.28 & 5.98 \\
Dice & 91.42 & 7.02 & 1.30 & 0.13 & 0.13 \\
Casino & 94.80 & 4.03 & 1.04 & 0.13 & 0.00 \\
Scratch cards & 51.63 & 33.03 & 9.62 & 5.07 & 0.65 \\
Lotteries & 76.98 & 17.04 & 4.68 & 1.04 & 0.26 \\
Bingo & 84.79 & 11.44 & 2.99 & 0.65 & 0.13 \\
Slot machines & 89.08 & 6.76 & 2.86 & 0.65 & 0.65 \\
Skill games & 71.91 & 15.60 & 5.59 & 4.55 & 2.34
\end{tabular}

within-subjects contrasts revealed significant interaction effects between FDIS dimensions and gender $\left[F_{(1,1004)}=55.20 ; p<\right.$ $\left.0.001 ; \eta_{\mathrm{p}}^{2}=0.052\right]$, age $\left[F_{(1,1004)}=6.67 ; p<0.01 ; \eta_{\mathrm{p}}^{2}=0.007\right]$, and SOGS-RA classification $\left[F_{(3,1004)}=22.19 ; p<0.001\right.$; $\left.\eta_{\mathrm{p}}^{2}=0.062\right)$. Over and above gender and age effects, these results indicated that, in general, at-risk and problem gamblers were more impulsive than non-gamblers and non-problem gamblers. Of interest, non-gamblers and non-problem gamblers scored significantly higher on the functional scale than on the dysfunctional one, whereas at-risk and problem gamblers scored significantly lower on the functional impulsivity scale than on the dysfunctional impulsivity dimension.

As regards delay discounting performance, all participants showed higher $k$-values for smaller, compared to larger delayed rewards. All pair-wise differences in $k$ between reward magnitudes were highly reliable overall and within the four groups (all $p s<0.001$ ).

Choice behavior was analyzed using a $4 \times 3$ mixed-model ANOVA of group by magnitude (small, medium, and large). The analysis yielded significant main effects due to gender $\left[F_{(1,1004)}\right.$ $\left.=9.38 ; p<0.01 ; \eta_{\mathrm{p}}^{2}=0.009\right]$, age $\left[F_{(1,1004)}=18.42 ; p<0.001\right.$; $\left.\eta_{\mathrm{p}}^{2}=.018\right]$, and group $\left[F_{(3,1004)}=5.32 ; p<0.01 ; \eta_{\mathrm{p}}^{2}=0.016\right]$, indicating that males scored higher than females on the MCQ, delay discounting become steeper as a function of age, and at-risk and problem gamblers showed higher rates of delay discounting than did non-gamblers and non-problem gamblers.

Regarding CFC-14 scores, results of a $4 \times 2$ repeated measures ANOVA yielded a significant main effect of gender $\left(F_{(1,1004)}=7.69 ; p<0.01 ; \eta_{\mathrm{p}}^{2}=0.008\right]$, with males reporting higher scores on the Immediate subscale than females, as well as an interaction effect between SOGS-RA group and the two dimensions of the CFC-14 $\left[F_{(3,1004)}=30.27 ; p<0.001 ; \eta_{\mathrm{p}}^{2}=\right.$ 0.083 ], indicating that Immediate scores increase as a function of gambling severity, whereas Future scores decrease according to gambling involvement.

A $4 \times 3$ repeated measures ANOVA was also conducted on DASS-21 scores. Results indicated significant main effects due to gender $\left[F_{(1,1004)}=41.04 ; p<0.001 ; \eta_{\mathrm{p}}^{2}=0.039\right]$ and SOGS-RA group $\left[F_{(3,1004)}=31.39 ; p<0.001 ; \eta_{\mathrm{p}}^{2}=0.086\right]$, showing that females scored significantly higher than males on the three DASS21 dimensions, and that negative emotional states increase as a function of gambling severity.

Means and standard deviations by SOGS-RA group are presented in Table 3. To facilitate interpretation, descriptive statistics are reported for the untransformed variables.

To identify the potential predictors of gambling behavior and gambling-related problems, gender, age and scores on FDIS, MCQ, CFC-14, and DASS-21 scales were input to a multiple regression analysis with SOGS-RA scores as the dependent measure. Results of hierarchical regression analysis (see Table 4) showed that, along with gender and age, dysfunctional impulsivity, anxiety, depression, short time horizon, and delay discounting significantly predicted gambling severity. The overall model explained about a third part of the total variance of the SOGS-RA $\left[R_{\text {adj }}^{2}=0.273 ; F_{(8,1001)}=48.35\right.$; $p<0.001]$. 
TABLE 3 | Means and standard deviations by SOGS-RA groups.

\begin{tabular}{|c|c|c|c|c|c|c|c|c|c|c|}
\hline & \multicolumn{2}{|c|}{ Non-gamblers } & \multicolumn{2}{|c|}{ Non-problem gamblers } & \multicolumn{2}{|c|}{ At-risk gamblers } & \multicolumn{2}{|c|}{ Problem gamblers } & \multicolumn{2}{|c|}{ Total sample } \\
\hline & Mean & $S D$ & Mean & $S D$ & Mean & $S D$ & Mean & $S D$ & Mean & $S D$ \\
\hline \multicolumn{11}{|l|}{ FDIS } \\
\hline Functional Impulsivity & 30.15 & 6.07 & 31.52 & 5.94 & 32.46 & 6.84 & 33.69 & 6.43 & 31.57 & 6.25 \\
\hline Dysfunctional Impulsivity & 28.68 & 6.75 & 30.12 & 6.87 & 34.10 & 7.01 & 36.71 & 7.58 & 31.09 & 7.34 \\
\hline \multicolumn{11}{|l|}{ MCQ } \\
\hline k Total score (overall k) & 0.02 & 0.01 & 0.02 & 0.02 & 0.03 & 0.03 & 0.04 & 0.04 & 0.02 & 0.02 \\
\hline k Small & 0.03 & 0.04 & 0.04 & 0.05 & 0.05 & 0.06 & 0.06 & 0.07 & 0.04 & 0.05 \\
\hline k Medium & 0.02 & 0.03 & 0.03 & 0.03 & 0.03 & 0.04 & 0.05 & 0.06 & 0.03 & 0.03 \\
\hline k Large & 0.01 & 0.01 & 0.02 & 0.02 & 0.02 & 0.04 & 0.03 & 0.04 & 0.02 & 0.03 \\
\hline \multicolumn{11}{|l|}{ CFC-14 } \\
\hline Total score & 4.88 & 0.58 & 4.76 & 0.66 & 4.46 & 0.78 & 4.11 & 0.90 & 4.68 & 0.72 \\
\hline Immediate & 19.86 & 6.30 & 20.41 & 6.39 & 22.96 & 7.54 & 25.13 & 8.52 & 21.15 & 6.97 \\
\hline Future & 32.17 & 6.85 & 31.03 & 6.94 & 29.46 & 8.74 & 26.60 & 8.01 & 30.63 & 7.52 \\
\hline \multicolumn{11}{|l|}{ DASS-21 } \\
\hline Total score & 14.79 & 11.16 & 18.83 & 12.42 & 22.24 & 13.63 & 27.75 & 14.54 & 19.31 & 13.03 \\
\hline Depression & 4.97 & 4.43 & 6.14 & 4.76 & 7.46 & 5.36 & 9.30 & 5.51 & 6.39 & 5.01 \\
\hline Anxiety & 4.29 & 4.05 & 4.95 & 4.40 & 6.14 & 4.70 & 7.94 & 5.40 & 5.27 & 4.57 \\
\hline Stress & 5.53 & 4.37 & 7.74 & 4.91 & 8.65 & 5.10 & 10.51 & 5.37 & 7.65 & 5.05 \\
\hline
\end{tabular}

Descriptive statistics are reported for the untransformed variables.

Finally, to analyze the developmental trajectories of gambling involvement, trait impulsivity, delay discounting, time perspective, and negative affective states, participants were divided into four age-groups (12-13, 14-15, 16-17, and 18-19 years, respectively). Subsequently, SOGS-RA scores across age-groups were analyzed by means of univariate ANOVA, whereas scores on the FDIS, MCQ, CFC-14, and DASS-21 subscales (within-participants variables) were submitted to repeated measures ANOVAs followed by Bonferroni post-hoc test, with gender, age group, and SOGS-RA classification as between-participants variables.

As far as SOGS-RA scores, a 2 (gender) $\times 4$ (age group) ANOVA yielded significant main effects of gender $\left[F_{(1,1002)}=\right.$ 77.66; $\left.p<0.001 ; \eta_{\mathrm{p}}^{2}=0.072\right]$ and age group $\left[F_{(3,1002)}=8.24\right.$; $\left.p<0.001 ; \eta_{\mathrm{p}}^{2}=0.024\right]$, showing that gambling severity varies as a function of gender, with males reporting higher scores than females, and increases progressively with age (see Figure 1).

In regard to functional and dysfunctional impulsivity, mixed model ANOVA, with gender, age group, and SOGS-RA group entered as between-subjects factors, and FDIS subscales as within-subjects factor, yielded a main effect due to SOGS-RA classification $\left[F_{(3,979)}=21.37 ; p<0.001 ; \eta_{\mathrm{p}}^{2}=0.061\right]$, indicating that both functional and dysfunctional impulsivity increased as a function of gambling severity. Furthermore, analysis yielded a significant interaction effect between gender and SOGS-RA classification $\left[F_{(3,979)}=2.97 ; p<0.05 ; \eta_{\mathrm{p}}^{2}=0.009\right]$, revealing that among non-gamblers females were less impulsive than males, whereas among problem gamblers females were more impulsive than males.

With regard to delay discounting, repeated measures ANOVA showed significant main effects of age group $\left[F_{(3,979)}=4.30\right.$; $\left.p<0.01 ; \eta_{\mathrm{p}}^{2}=0.013\right]$ and SOGS-RA group $\left[F_{(3,979)}=2.81 ; p\right.$ $<0.05 ; \eta_{\mathrm{p}}^{2}=0.009$ ], indicating that scores increased with age and as a function of gambling severity.

With respect to time perspective, no significant betweensubjects effect was observed. However, within-subjects contrasts revealed a significant interaction of CFC-14 scores and SOGS-RA classification $\left[F_{(1,979)}=15.35 ; p<0.001 ; \eta_{\mathrm{p}}^{2}=0.045\right]$, reflecting the fact that Immediate scores increased, whereas Future scores decreased according to gambling severity.

As far as negative affectivity, mixed-model ANOVA identified significant main effects of gender $\left[F_{(1,979)}=9.27 ; p<0.01 ; \eta_{\mathrm{p}}^{2}=\right.$ 0.009 ], with females obtaining higher DASS-21 scores than males, and SOGS-RA classification $\left[F_{(3,979)}=18.44 ; p<0.001 ; \eta_{\mathrm{p}}^{2}=\right.$ 0.053 , indicating that negative emotional states increased as a function of gambling severity.

Taken together, these results indicated that gambling severity contributes more than age in shaping the developmental trajectories of functional and dysfunctional impulsivity, delay discounting, time perspective, and negative affective states.

\section{DISCUSSION}

The present study is the first research that analyzes the interplay of self-reported functional and dysfunctional impulsivity, delay discounting, time perspective, and emotional negative states to gambling severity in adolescents. Previous research suggest the idea that problematic gambling, impulsivity, shortsightedness, and negative psychological states in adolescence are somewhat nested. However, and this is the novelty of our paper, to date no study had ever considered these constructs jointly. 
TABLE 4 | Summary of hierarchical regression analysis.

\begin{tabular}{|c|c|c|c|c|c|c|c|}
\hline Variable & B & $R^{2}$ & $\Delta R^{2}$ & $\beta$ & $t$ & $p$ & VIF \\
\hline \multicolumn{8}{|l|}{ STEP 1} \\
\hline Gender & -0.258 & 0.087 & 0.087 & -0.270 & -8.924 & 0.000 & 1.007 \\
\hline Age & 0.034 & & & 0.145 & 4.792 & 0.000 & 1.007 \\
\hline \multicolumn{8}{|l|}{ STEP 2} \\
\hline Gender & -0.267 & 0.193 & 0.106 & -0.279 & -9.827 & 0.000 & 1.008 \\
\hline Age & 0.032 & & & 0.139 & 4.885 & 0.000 & 1.007 \\
\hline $\begin{array}{l}\text { Dysfunctional } \\
\text { Impulsivity }\end{array}$ & 0.021 & & & 0.326 & 11.510 & 0.000 & 1.001 \\
\hline \multicolumn{8}{|l|}{ STEP 3} \\
\hline Gender & -0.289 & 0.230 & 0.036 & -0.302 & -10.805 & 0.000 & 1.022 \\
\hline Age & 0.030 & & & 0.129 & 4.637 & 0.000 & 1.010 \\
\hline $\begin{array}{l}\text { Dysfunctional } \\
\text { Impulsivity }\end{array}$ & 0.018 & & & 0.281 & 9.880 & 0.000 & 1.057 \\
\hline DASS-21 Anxiety & 0.021 & & & 0.198 & 6.893 & 0.000 & 1.076 \\
\hline \multicolumn{8}{|l|}{ STEP 4} \\
\hline Gender & -0.289 & 0.250 & 0.020 & -0.302 & -10.920 & 0.000 & 1.022 \\
\hline Age & 0.029 & & & 0.123 & 4.487 & 0.000 & 1.011 \\
\hline $\begin{array}{l}\text { Dysfunctional } \\
\text { Impulsivity }\end{array}$ & 0.016 & & & 0.246 & 8.524 & 0.000 & 1.117 \\
\hline DASS-21 Anxiety & 0.022 & & & 0.208 & 7.318 & 0.000 & 1.081 \\
\hline CFC-14 Future & -0.009 & & & -0.146 & -5.176 & 0.000 & 1.059 \\
\hline \multicolumn{8}{|l|}{ STEP 5} \\
\hline Gender & -0.268 & 0.264 & 0.014 & -0.281 & -10.091 & 0.000 & 1.054 \\
\hline Age & 0.030 & & & 0.127 & 4.663 & 0.000 & 1.012 \\
\hline $\begin{array}{l}\text { Dysfunctional } \\
\text { Impulsivity }\end{array}$ & 0.013 & & & 00.199 & 6.492 & 0.000 & 1.278 \\
\hline DASS-21 Anxiety & 0.020 & & & 0.195 & 6.880 & 0.000 & 1.093 \\
\hline CFC-14 Future & -0.010 & & & -0.160 & -5.701 & 0.000 & 1.074 \\
\hline $\begin{array}{l}\text { CFC-14 } \\
\text { Immediate }\end{array}$ & 0.009 & & & 0.130 & 4.367 & 0.000 & 1.210 \\
\hline \multicolumn{8}{|l|}{ STEP 6} \\
\hline Gender & -0.271 & 0.272 & 0.008 & -0.283 & -10.238 & 0.000 & 1.055 \\
\hline Age & 0.028 & & & 0.120 & 4.414 & 0.000 & 1.018 \\
\hline $\begin{array}{l}\text { Dysfunctional } \\
\text { Impulsivity }\end{array}$ & 0.012 & & & 0.186 & 6.052 & 0.000 & 1.299 \\
\hline DASS-21 Anxiety & 0.012 & & & 0.115 & 3.146 & 0.000 & 1.853 \\
\hline CFC-14 Future & -0.010 & & & -0.164 & -5.873 & 0.000 & 1.076 \\
\hline $\begin{array}{l}\text { CFC-14 } \\
\text { Immediate }\end{array}$ & 0.009 & & & 0.127 & 4.273 & 0.000 & 1.212 \\
\hline $\begin{array}{l}\text { DASS-21 } \\
\text { Depression }\end{array}$ & 0.012 & & & 0.125 & 3.382 & 0.001 & 1.876 \\
\hline \multicolumn{8}{|l|}{ STEP 7} \\
\hline Gender & -0.260 & 0.280 & 0.008 & -0.272 & -9.808 & 0.000 & 1.071 \\
\hline Age & 0.025 & & & 0.105 & 3.840 & 0.000 & 1.047 \\
\hline $\begin{array}{l}\text { Dysfunctional } \\
\text { Impulsivity }\end{array}$ & 0.012 & & & 0.186 & 6.100 & 0.000 & 1.299 \\
\hline DASS-21 Anxiety & 0.011 & & & 0.108 & 2.939 & 0.003 & 1.862 \\
\hline CFC-14 Future & -0.010 & & & -0.156 & -5.600 & 0.000 & 1.084 \\
\hline $\begin{array}{l}\text { CFC-14 } \\
\text { Immediate }\end{array}$ & 0.008 & & & 0.118 & 3.984 & 0.000 & 1.222 \\
\hline $\begin{array}{l}\text { DASS-21 } \\
\text { Depression }\end{array}$ & 0.012 & & & 0.129 & 3.521 & 0.000 & 1.879 \\
\hline$k$ Total score & 0.037 & & & 0.090 & 3.236 & 0.001 & 1.066 \\
\hline
\end{tabular}

$B$, unstandardized coefficient; $\Delta R^{2}, R$ square change; $\beta$, standardized regression coefficient; VIF, variance inflation factor.
On the whole, data from this study indicated that, relative to non-gamblers and non-problem gamblers, at-risk and problem gamblers showed higher levels of impulsivity, steeper delay discounting, shorter time horizon, and reported experiencing significantly higher levels of depression, anxiety, and stress.

In line with previous studies (van den Bos et al., 2013; ScholesBalog et al., 2014; Welte et al., 2015; Raylu et al., 2016; for reviews see also Johansson et al., 2009; Griffiths, 2011; Donati et al., 2013), gender showed a significant negative relationship with SOGSRA scores. However, no gender difference was observed with respect to the modes of gambling activities (offline vs. online). Interestingly, gambling routes did not vary as a function of age. Generally speaking, notwithstanding the advent of internet gambling, participants appeared to prefer traditional routes of gambling, probably because online gambling requires a credit card. However, especially striking is the amount of time and money spent on gambling activities. Just consider that a quarter of adolescent problem gamblers reported wasting between 10 and 50 Euros, and $14.5 \%$ of them more than 50 Euros in one day. In our opinion, future research should ask participants how they raise funds.

As far as impulsivity, results further support previous studies demonstrating that impulsivity, apart from the instruments used to asses it, remains one of the most robust feature associated with disordered gambling (MacKillop et al., 2014). What we first observed on adolescents dovetails with Maccallum et al. (2007), who found that, compared to normative data, adult pathological gamblers seeking treatment reported higher scores on both functional and dysfunctional impulsivity. More interestingly, our results showed that only dysfunctional impulsivity represents a significant predictor of severity of adolescent gambling involvement.

As regards to delay discounting, the results are in accordance with previous research demonstrating that pathological gamblers devalue or discount delayed rewards to a greater extent than nongamblers and non-problem gamblers do (Petry and Casarella, 1999; e.g., Alessi and Petry, 2003; Madden et al., 2011; Michalczuk et al., 2011; Brevers et al., 2012; Miedl et al., 2012; Petry, 2012; Kräplin et al., 2014; see also Gray and MacKillop, 2014; Cosenza and Nigro, 2015; for a review see Wiehler and Peters, 2015; Cosenza et al., 2016; Nigro and Cosenza, 2016; Ciccarelli et al., 2016b).

In light of our results, adolescent gamblers show a similar shortsightedness by ignoring the future consequences of their actual behavior. More specifically, at-risk and problem gamblers appear to be more prone to focus on the immediate outcomes of their behavior than both non-gamblers and non-problem gamblers. This finding extends evidence obtained on both adult and adolescent samples (Hodgins and Engel, 2002; Whiteside et al., 2005; Toplak et al., 2007; Daugherty and Brase, 2010; MacLaren et al., 2012; Cosenza et al., 2014, 2016; MacKillop et al., 2014; Cosenza and Nigro, 2015; Ciccarelli et al., 2016a).

Since dysfunctional impulsivity was found to be strongly associated with the tendency to ignore hard facts when making decision (Dickman, 1990), it is no wonder that there are significant correlations among SOGS-RA, FDIS, and both CFC-14 and MCQ scores. It may be that high levels of 


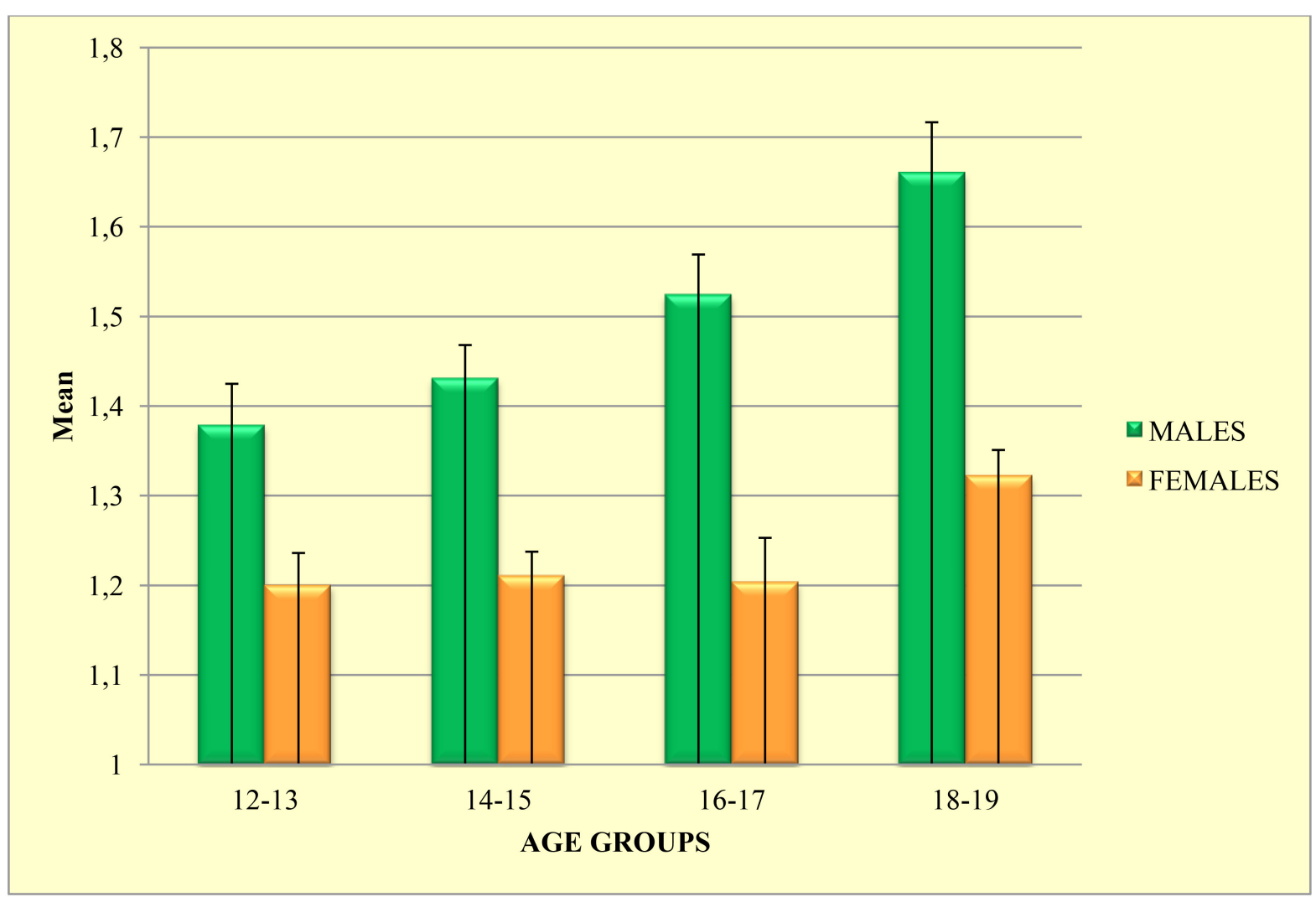

FIGURE 1 | SOGS-RA mean scores as a function of gender and age. Error bars indicate standard error of the mean.

dysfunctional impulsivity exacerbate the individual's inability to consider carefully the long-term future consequences of actions and to pay attention to one's own future, with all these impulsivity facets concurring to foster gambling addiction.

As with previous research (Lee et al., 2011; Hartmann and Blaszczynski, 2016; for reviews see Ciccarelli et al., 2017), the present study found that the more individuals have a problematic gambling involvement, the more they experience anxiety and depression. These results confirm the findings of previous studies demonstrating that among both adolescents and adults anxiety and depression co-occur with problematic gambling (Blaszczynski and McConaghy, 1989; Coman et al., 1997; Raylu and Oei, 2002; Kim et al., 2006; Barrault and Varescon, 2013; Martin et al., 2014; Estevez et al., 2015; Chinneck et al., 2016; Cunningham et al., 2016; Toneatto and Pillai, 2016; see also, Takamatsu et al., 2016). It may be that depression foregoes problem gambling, which serves to relieve negative emotions and to avoid problems (Blaszczynski and Nower, 2002) or that problematic gambling involvement increasingly leads to depressive symptoms due to the consequent social isolation and money problems (Dussault et al., 2011). Although it is difficult to determine whether anxiety and depression are primary, secondary, or concurrent with gambling, recently Raylu et al. (2016) have demonstrated that negative affectivity directly predicts gambling behavior.
Results of cross-sectional analyses indicated that gambling involvement increases as a function of gender and age. As depicted in Figure 1, the gambling involvement increases linearly with age among males, whereas among females the trend remains quite flat from 12 to 17 years, but picks significantly in late adolescence. This result corroborates the existence of a telescoping phenomenon, "whereby women as compared to men begin engagement in the behavior on average later in life than do men but the time between initial participation and development of a problem is shorter (or telescoped) in women as compared to men” (Potenza, 2013, p. S26).

As far as impulsivity, results indicated that the developmental trajectories of functional and dysfunctional impulsivity among adolescents are shaped mostly by the severity of gambling involvement. The same holds true for time perspective and delay discounting. Indeed, adolescent at-risk and problem gamblers appeared to devote less attention to the future, with more of the focus on the present (for similar results see Toplak et al., 2007; Cosenza and Nigro, 2015), and to have a weak orientation to the future also by choosing smaller but immediate rewards over larger but delayed rewards. Although some cross-sectional studies have demonstrated that in healthy adolescents delay discounting slightly declines in late adolescence (e.g., Green et al., 1994; Olson et al., 2007; Steinberg et al., 2009; see Albert and Steinberg, 2011 for a review), the results of cross-sectional 
analysis might suggest that gambling severity put the positive age-related changes across adolescence almost in the shade.

Finally, the results indicate that negative psychological states, namely anxiety and depression, increase as a function of gender and gambling involvement. These findings further support previous research reporting a stronger association between gambling severity and both depression and anxiety disorders in women than in men (Getty et al., 2000; Petry et al., 2005; SteMarie et al., 2006; Kessler et al., 2008; e.g., Desai and Potenza, 2008; Williams et al., 2012; see also Cunningham et al., 2016).

Given that anxiety and depression have been considered both precursors and consequences of problem gambling (see Hartmann and Blaszczynski, 2016), having found that female adolescents reported significantly greater levels of anxiety and depression suggests that gambling research, prevention, and treatment programs should consider carefully gender differences. In addition, since the combination of high impulsivity and emotional vulnerability contributes to foster the cycle of pathological gambling (e.g., McCormick et al., 1984), treatment protocols for gambling disorder should also take in account this underlying interplay. Indeed, as stressed by Blaszczynski and Nower (2002) and Hartmann and Blaszczynski (2016), the cooccurrence of emotional vulnerability and problematic gambling makes treatment more difficult. If this is true for adults, it is especially true for adolescents.

\section{LIMITATIONS}

Although there are several strengths of the present study, including the large sample of participants, there are some limitations that should be considered when interpreting the present results. First, the current data are mainly based on selfreport measures. In addition, it is to bear in mind that some authors questioned the validity of SOGS-RA (see Stinchfield, 2010 for a review), whereas other authors support the suitability of the instrument as a screening tool in adolescent populations (see Chiesi et al., 2013). Besides, it is worth to specify that the findings obtained are based on the general population of adolescents (12-19 years old), since no clinical group has been included in the study. Secondly, even if several studies demonstrated that there is no difference across hypothetical and potentially real rewards (e.g., Johnson and Bickel, 2002; Madden et al., 2003; Lagorio and Madden, 2005), delay discounting was evaluated using a behavioral measure that relies on hypothetical monetary choices. A final limitation is the use of cross-sectional sampling to analyze the developmental trajectories of gambling involvement, functional, and dysfunctional impulsivity, delay discounting, consideration of future consequences, and negative affectivity instead of a more appropriate longitudinal approach. Despite these limitations, to the authors' knowledge, the present study is the first to investigate the interplay of functional and dysfunctional impulsivity, time perspective, delay discounting, and negative affectivity on gambling severity among adolescents.

\section{AUTHOR CONTRIBUTIONS}

Authors MC and GN together designed the study and wrote the protocol. Author MCi conducted literature searches and provided summaries of previous research studies. Author GN conducted the statistical analysis. Authors MC and GN wrote the first draft of the manuscript and all authors contributed to and have approved the final manuscript.

\section{REFERENCES}

Ainslie, G. (1975). Specious reward: a behavioral theory of impulsiveness and impulse control. Psychol. Bull. 82, 463-496. doi: 10.1037/h0076860

Albert, D., and Steinberg, L. (2011). Judgment and decision making in adolescence. J. Res. Adolesc. 21, 211-224. doi: 10.1111/j.1532-7795.2010.00724.x

Alessi, S. M., and Petry, N. M. (2003). Pathological gambling severity is associated with impulsivity in a delay discounting procedure. Behav. Process 64, 345-354. doi: 10.1016/S0376-6357(03)00150-5

Amlung, M., and MacKillop, J. (2011). Delayed reward discounting and alcohol misuse: the roles of response consistency and reward magnitude. J. Exp. Psychopathol. 2, 418-431. doi: 10.5127/jep.017311

Barrault, S., and Varescon, I. (2013). Cognitive distortions, anxiety, and depression among regular and pathological gambling online poker players. Cyberpsychol. Behav. Soc. Netw. 16, 183-188. doi: 10.1089/cyber.2012.0150

Blaszczynski, A., and McConaghy, N. (1989). Anxiety and/or depression in the pathogenesis of addictive gambling. Subst. Use Misuse 24, 337-350. doi: 10.3109/10826088909047292

Blaszczynski, A., and Nower, L. (2002). A pathways model of problem and pathological gambling. Addiction 97, 487-499. doi: 10.1046/j.1360-0443.2002.00015.x

Blinn-Pike, L., Worthy, S. L., and Jonkman, J. N. (2010). Adolescent gambling: a review of an emerging field of research. J. Adolesc. Health 47, 223-236. doi: 10.1016/j.jadohealth.2010.05.003

Bottesi, G., Ghisi, M., Altoè, G., Conforti, E., Melli, G., and Sica, C. (2015). The Italian version of the depression anxiety stress scales-21: factor structure and

psychometric properties on community and clinical samples. Compr. Psychiat. 60, 170-181. doi: 10.1016/j.comppsych.2015.04.005

Brevers, D., Cleeremans, A., Verbruggen, F., Bechara, A., Kornreich, C., Verbanck, P., et al. (2012). Impulsive action but not impulsive choice determines problem gambling severity. PLoS ONE 7:e50647. doi: 10.1371/journal.pone.0050647

Calado, F., Alexandre, J., and Griffiths, M. D. (2016). Prevalence of adolescent problem gambling: A systematic review of recent research. J. Gambl. Stud. doi: 10.1007/s10899-016-9627-5. [Epub ahead of print].

Chiesi, F., Donati, M. A., Galli, S., and Primi, C. (2013). The suitability of the South Oaks Gambling Screen Revised for Adolescents (SOGS-RA) as a screening tool: IRT-based evidence. Psychol. Addict. Behav. 27, 287-293. doi: 10.1037/a0029987

Chinneck, A., Mackinnon, S. P., and Stewart, S. H. (2016). Investigating possible reciprocal relations between depressive and problem gambling symptoms in emerging adults. Can. J. Psychiat. 61, 93-101. doi: 10.1177/0706743715625934

Ciccarelli, M., Griffiths, M. D., Nigro, G., and Cosenza, M. (2016a). Decisionmaking, cognitive distortions and alcohol use in adolescent problem and nonproblem gamblers: an experimental study. J. Gambl. Stud. 32, 1203-1213. doi: 10.1007/s10899-016-9597-7

Ciccarelli, M., Griffiths, M. D., Nigro, G., and Cosenza, M. (2017). Decision making, cognitive distortions and emotional distress: a comparison between pathological gamblers and healthy controls. J. Behav. Ther. Exp. Psychiatry 54, 204-210. doi: 10.1016/j.jbtep.2016.08.012

Ciccarelli, M., Malinconico, R., Griffiths, M. D., Nigro, G., and Cosenza, M. (2016b). Reward preferences of pathological gamblers under conditions of uncertainty: an experimental study. J. Gambl. Stud. 32, 1175-1184. doi: 10.1007/s10899-016-9593-y 
Colasante, E., Gori, M., Bastiani, L., Scalese, M., Siciliano, V., and Molinaro, S. (2013). Italian adolescent gambling behaviour: psychometric evaluation of the South Oaks Gambling Screen: Revised for Adolescents (SOGSRA) among a sample of Italian students. J. Gambl. Stud. 30, 789-801. doi: $10.1007 /$ s10899-013-9385-6

Coman, G. J., Burrows, G. D., and Evans, B. J. (1997). Stress and anxiety as factors in the onset of problem gambling: implications for treatment. Stress Med. 13, 235-244. doi: 10.1002/(SICI)1099-1700(199710)13:4<235::AID-SMI748>3.0.CO;2-4

Cosenza, M., Baldassarre, I., Matarazzo, O., and Nigro, G. (2014). Youth at stake: alexithymia, cognitive distortions, and problem gambling in late adolescents. Cognit. Comput. 6, 652-660. doi: 10.1007/s12559-014-9274-z

Cosenza, M., Griffiths, M. D., Nigro, G., and Ciccarelli, M. (2016). Risktaking, delay discounting, and time perspective in adolescent gamblers: an experimental study. J. Gambl. Stud. doi: 10.1007/s10899-016-9623-9. [Epub ahead of print].

Cosenza, M., and Nigro, G. (2015). Wagering the future: cognitive distortions, impulsivity, delay discounting, and time perspective in adolescent gambling. J. Adolesc. 45, 56-66. doi: 10.1016/j.adolescence.2015.08.015

Cunningham, J. A., Hodgins, D. C., Bennett, K., Bennett, A., Talevski, M., Mackenzie, C. S., et al. (2016). Online interventions for problem gamblers with and without co-occurring mental health symptoms: protocol for a randomized controlled trial. BMC Public Health 16:624. doi: 10.1186/s12889-016-3291-7

Daugherty, J. R., and Brase, G. L. (2010). Taking time to be healthy: predicting health behaviors with delay discounting and time perspective. Pers. Individ. Dif. 48, 202-207. doi: 10.1016/j.paid.2009.10.007

Delfabbro, P., King, D., and Derevensky, J. (2016). Adolescent gambling and problem gambling: prevalence, current issues and concerns. Curr. Addict. Rep. 3, 268-274. doi: 10.1007/s40429-016-0105-Z

Desai, R. A., and Potenza, M. N. (2008). Gender differences in the associations between past-year gambling problems and psychiatric disorders. Soc. Psychiatry Psychiatr. Epidemiol. 43, 173-183. doi: 10.1007/s00127-007-0283-Z

Dickman, S. J. (1990). Functional and dysfunctional impulsivity: personality and cognitive correlates. J. Pers. Soc. Psychol. 58, 95-102. doi: 10.1037/0022-3514.58.1.95

Donati, M. A., Chiesi, F., and Primi, C. (2013). A model to explain atrisk/problem gambling among male and female adolescents: gender similarities and differences. J. Adolesc. 36, 129-137. doi: 10.1016/j.adolescence.2012. 10.001

Dowling, N. A., Cowlishaw, S., Jackson, A. C., Merkouris, S. S., Francis, K. L., and Christensen, D. R. (2015). Prevalence of psychiatric co-morbidity in treatmentseeking problem gamblers: a systematic review and meta-analysis. Aust. N. Z. J. Psychiatry 49, 519-539. doi: 10.1177/0004867415575774

Dussault, F., Brendgen, M., Vitaro, F., Wanner, B., and Tremblay, R. E. (2011). Longitudinal links between impulsivity, gambling problems and depressive symptoms: a transactional model from adolescence to early adulthood. J. Child Psychol. Psychiatry 52, 130-138. doi: 10.1111/j.1469-7610.2010.02313.x

El-Guebaly, N., Patten, S. B., Currie, S., Williams, J. V., Beck, C. A., Maxwell, C. J., et al. (2006). Epidemiological associations between gambling behavior, substance use and mood and anxiety disorders. J. Gambl. Stud. 22, 275-287. doi: 10.1007/s10899-006-9016-6

Estevez, A., Herrero-Fernández, D., Sarabia, I., and Jauregui, P. (2015). The impulsivity and sensation-seeking mediators of the psychological consequences of pathological gambling in adolescence. J. Gambl. Stud. 31, 91-103. doi: 10.1007/s10899-013-9419-0

Gainsbury, S. M., Delfabbro, P., King, D. L., and Hing, N. (2016a). An exploratory study of gambling operators' use of social media and the latent messages conveyed. J. Gambl. Stud. 32, 125-141. doi: 10.1007/s10899-015-9525-2

Gainsbury, S. M., King, D. L., Russell, A. M., Delfabbro, P., Derevensky, J., and Hing, N. (2016b). Exposure to and engagement with gambling marketing in social media: reported impacts on moderate-risk and problem gamblers. Psychol. Addict. Behav. 30, 268-274. doi: 10.1037/adb0000156

Gee, P., Coventry, K. R., and Birkenhead, D. (2005). Mood state and gambling: using mobile telephones to track emotions. Br. J. Psychol. 96, 53-66. doi: 10.1348/000712604X15536

Getty, H. A., Watson, J., and Frisch, G. R. (2000). A comparison of depression and styles of coping in male and female GA members and controls. J. Gambl. Stud. 16, 377-391. doi: 10.1023/A:1009480106531
Gray, J. C., and MacKillop, J. (2014). Genetic basis of delay discounting in frequent gamblers: Examination of a priori candidates and exploration of a panel of dopamine-related loci. Brain Behav. 4, 812-821. doi: 10.1002/brb3.284

Green, L., Fisher, E. B. Jr., Perlow, S., and Sherman, L. (1981). Preference reversal and self control: choice as a function of reward amount and delay. Behav. Anal. Lett. 1, 43-51.

Green, L., Fry, A., and Myerson, J. (1994). Discounting of delayed rewards: a life-span comparison. Psychol. Sci. 5, 33-36. doi: 10.1111/j.1467-9280.1994.tb00610.x

Griffiths, M. (1995). The role of subjective mood states in the maintenance of fruit machine gambling behaviour. J. Gambl. Stud. 11, 123-135. doi: 10.1007/BF02107111

Griffiths, M. D. (2011). “Adolescent gambling," in Encyclopedia of Adolescence, Vol. 3, eds B. B. Bradford and M. Prinstein (San Diego, CA: Academic Press), 11-20. doi: 10.1016/B978-0-12-373951-3.00113-7

Gupta, R., and Derevensky, J. (2014). Reflections on underage gambling. Responsible Gambl. Rev. 1, 37-50.

Hartmann, M., and Blaszczynski, A. (2016). The longitudinal relationships between psychiatric disorders and gambling disorders. Int. J. Ment. Health Addict. doi: 10.1007/s11469-016-9705-z. [Epub ahead of print].

Hodgins, D. C., and Engel, A. (2002). Future time perspective in pathological gamblers. J. Nerv. Ment. Dis. 190, 775-780. doi: 10.1097/00005053-200211000-00008

Holt, D. D., Green, L., and Myerson, J. (2003). Is discounting impulsive? Evidence from temporal and probability discounting in gambling and non-gambling college students. Behav. Process 64, 355-367. doi: 10.1016/S0376-6357(03)00141-4

Johansson, A., Grant, J. E., Kim, S. W., Odlaug, B. L., and GØtestam, K. G. (2009). Risk factors for problematic gambling: a critical literature review. J. Gambl. Stud. 25, 67-92. doi: 10.1007/s10899-008-9088-6

Johnson, M. W., and Bickel, W. K. (2002). Within-subject comparison of real and hypothetical money rewards in delay discounting. J. Exp. Anal. Behav. 77, 129-146. doi: 10.1901/jeab.2002.77-129

Joireman, J., Shaffer, M. J., Balliet, D., and Strathman, A. (2012). Promotion orientation explains why future-oriented people exercise and eat healthy: evidence from the two-factor consideration of future consequences-14 scale. J. Pers. Soc. Psychol. 38, 1272-1287. doi: 10.1177/0146167212449362

Kessler, R. C., Hwang, I., LaBrie, R., Petukhova, M., Sampson, N. A., Winters, K. C., et al. (2008). DSM-IV pathological gambling in the national comorbidity survey replication. Psychol. Med. 38, 1351-1360. doi: 10.1017/S0033291708002900

Kim, S. W., Grant, J. E., Eckert, E. D., Faris, P. L., and Hartman, B. K. (2006). Pathological gambling and mood disorders: Clinical associations and treatment implications. J. Affect. Disord. 92, 109-116. doi: 10.1016/j.jad.2005.12.040

Kirby, K. N., and Marakovic, N. N. (1996). Delay-discounting probabilistic rewards. Rates decrease as amounts increase. Psychon. B. Rev. 3, 100-104. doi: 10.3758/BF03210748

Kirby, K. N., Petry, N. M., and Bickel, W. K. (1999). Heroin addicts have higher discount rates for delayed reward than non-drug-using controls. J. Exp. Psychol. Gen. 128, 78-87. doi: 10.1037/0096-3445.128.1.78

Kräplin, A., Dshemuchadse, M., Behrendt, S., Scherbaum, S., Goschke, T., and Bühringer, G. (2014). Dysfunctional decision-making in pathological gambling: pattern specificity and the role of impulsivity. Psychiatry Res. 215, 675-682. doi: 10.1016/j.psychres.2013.12.041

Ladouceur, R., Goulet, A., and Vitaro, F. (2013). Prevention programmes for youth gambling: a review of the empirical evidence. Int. Gamb. Stud. 13, 141-159. doi: 10.1080/14459795.2012.740496

Ladouceur, R., Sylvain, C., Sevigny, S., Poirier, L., Brisson, L., Dias, C., et al. (2006). Pathological gamblers: Inpatients' versus outpatients' characteristics. J. Gambl. Stud. 22, 443-450. doi: 10.1007/s10899-006-9022-8

Lagorio, C. H., and Madden, G. J. (2005). Delay discounting of real and hypothetical rewards III: steady-state assessments, forced-choice trials, and all real rewards. Behav. Process 69, 173-187. doi: 10.1016/j.beproc.2005.02.003

Lee, G. P., Storr, C. L., Ialongo, N. S., and Martins, S. S. (2011). Compounded effect of early adolescence depressive symptoms and impulsivity on late adolescence gambling: a longitudinal study. J. Adolesc. Health 48, 164-169. doi: 10.1016/j.jadohealth.2010.06.002

Lorains, F. K., Cowlishaw, S., and Thomas, S. A. (2011). Prevalence of comorbid disorders in problem and pathological gambling: systematic 
review and meta-analysis of population surveys. Addiction 106, 490-498. doi: 10.1111/j.1360-0443.2010.03300.x

Lorains, F. K., Dowling, N. A., Enticott, P. G., Bradshaw, J. L., Trueblood, J. S., and Stout, J. C. (2014). Strategic and non-strategic problem gamblers differ on decision-making under risk and ambiguity. Addiction 109, 1128-1137. doi: 10.1111/add.12494

Lovibond, P. F., and Lovibond, S. H. (1995). The structure of negative emotional states: Comparison of the Depression Anxiety Stress Scales (DASS) with the beck depression and anxiety inventories. Behav. Res. Ther. 33, 335-343. doi: 10.1016/0005-7967(94)00075-U

Maccallum, F., Blaszczynski, A., Ladouceur, R., and Nower, L. (2007). Functional and dysfunctional impulsivity in pathological gambling. Pers. Individ. Dif. 43, 1829-1838. doi: 10.1016/j.paid.2007.06.002

MacKillop, J., Anderson, E. J., Castelda, B. A., Mattson, R. E., and Donovick, P. J. (2006). Divergent validity of measures of cognitive distortions, impulsivity, and time perspective in pathological gambling. J. Gambl. Stud. 22, 339-354. doi: 10.1007/s10899-006-9021-9

MacKillop, J., Miller, J. D., Fortune, E., Maples, J., Lance, C. E., Campbell, W. K., et al. (2014). Multidimensional examination of impulsivity in relation to disordered gambling. Exp. Clin. Psychopharm. 22, 176-185. doi: $10.1037 / \mathrm{a} 0035874$

MacLaren, V. V., Fugelsang, J. A., Harrigan, K. A., and Dixon, M. J. (2012). Effects of impulsivity, reinforcement sensitivity, and cognitive style on pathological gambling symptoms among frequent slot machine players. Pers. Individ. Dif. 52, 390-394. doi: 10.1016/j.paid.2011.10.044

Madden, G. J., Begotka, A. M., Raiff, B. R., and Kastern, L. L. (2003). Delay discounting of real and hypothetical rewards. Exp. Clin. Psychopharm. 11, 139-145. doi: 10.1037/1064-1297.11.2.139

Madden, G. J., Francisco, M. T., Brewer, A. T., and Stein, J. S. (2011). Delay discounting and gambling. Behav. Process 87, 43-49. doi: 10.1016/j.beproc.2011.01.012

Madden, G. J., Petry, N. M., and Johnson, P. S. (2009). Pathological gamblers discount probabilistic rewards less steeply than matched controls. Exp. Clin. Psychopharm. 17, 283-290. doi: 10.1037/a0016806

Martin, R. J., Usdan, S., Cremeens, J., and Vail-Smith, K. (2014). Disordered gambling and co-morbidity of psychiatric disorders among college students: an examination of problem drinking, anxiety and depression. J. Gambl. Stud. 30, 321-333. doi: 10.1007/s10899-013-9367-8

Matthews, N., Farnsworth, B., and Griffiths, M. D. (2009). A pilot study of problem gambling among student online gamblers: mood states as predictors of problematic behavior. Cyberpsychol. Behav. 12, 741-745. doi: 10.1089/cpb.2009.0050

McCormack, A., Shorter, G. W., and Griffiths, M. D. (2014). An empirical study of gender differences in online gambling. J. Gambl. Stud. 30, 71-88. doi: $10.1007 /$ s10899-012-9341-x

McCormick, R. A., Russo, A. M., Ramirez, L. F., and Taber, J. I. (1984). Affective disorders among pathological gamblers seeking treatment. Am. J. Psychiatry 141, 215-218. doi: 10.1176/ajp.141.2.215

Messerlian, C., Byrne, A. M., and Derevensky, J. L. (2004). Gambling, youth and the internet: should we be concerned? Can. Child Adolesc. Psychiatr. Rev. 13, 3-6.

Messerlian, C., Derevensky, J., and Gupta, R. (2005). Youth gambling problems: a public health perspective. Health Promot. Internat. 20, 69-79. doi: 10.1093/heapro/dah509

Michalczuk, R., Bowden-Jones, H., Verdejo-Garcia, A., and Clark, L. (2011). Impulsivity and cognitive distortions in pathological gamblers attending the UK National problem gambling clinic: a preliminary report. Psychol. Med. 41, 2625-2635. doi: 10.1017/S003329171100095X

Miedl, S. F., Peters, J., and Büchel, C. (2012). Altered neural reward representations in pathological gamblers revealed by delay and probability discounting. Arch. Gen. Psychiatry 69, 177-186. doi: 10.1001/archgenpsychiatry.2011.1552

Nigro, G., and Cosenza, M. (2016). Living in the now: decision-making and delay discounting in adolescent gamblers. J. Gambl. Stud. 32, 1191-1202. doi: 10.1007/s10899-016-9595-9

Nigro, G., Cosenza, M., Ciccarelli, M., and Joireman, J. (2016). An Italian translation and validation of the consideration of future Consequences-14 scale. Pers. Individ. Dif. 101, 333-340. doi: 10.1016/j.paid.2016.06.014
Nower, L., and Blaszczynski, A. (2010). Gambling motivations, money-limiting strategies, and precommitment preferences of problem versus non-problem gamblers. J. Gamb. Stud. 26, 361-372. doi: 10.1007/s10899-009-9170-8

Nower, L., Gupta, R., Blaszczynski, A., and Derevensky, J. (2004). Suicidality and depression among youth gamblers: A preliminary examination of three studies. Int. Gambl. Stud. 4, 69-80. doi: 10.1080/1445979042000224412

Olason, D. T., Kristjansdottir, E., Einarsdottir, H., Haraldsson, H., Bjarnason, G., and Derevensky, J. L. (2011). Internet gambling and problem gambling among 13 to 18 year old adolescents in Iceland. Int. J. Ment. Health Addict. 9, 257-263. doi: 10.1007/s11469-010-9280-7

Olson, E. A., Hooper, C. J., Collins, P., and Luciana, M. (2007). Adolescents' performance on delay and probability discounting tasks: Contributions of age, intelligence, executive functioning, and self-reported externalizing behavior. Pers. Individ. Dif. 43, 1886-1897. doi: 10.1016/j.paid.2007.06.016

Pagani, L. S., Derevensky, J. L., and Japel, C. (2010). Does early emotional distress predict later child involvement in gambling? Can. J. Psychiat. 55, 507-513. doi: 10.1177/070674371005500805

Petry, N. M. (2012). Discounting of probabilistic rewards is associated with gambling abstinence in treatment-seeking pathological gamblers. J. Abnorm. Psychol. 121, 151-167. doi: 10.1037/a0024782

Petry, N. M., and Casarella, T. (1999). Excessive discounting of delayed rewards in substance abusers with gambling problems. Drug Alcohol Depend. 56, 25-32. doi: 10.1016/S0376-8716(99)00010-1

Petry, N. M., Stinson, F. S., and Grant, B. F. (2005). Comorbidity of DSM-IV pathological gambling and other psychiatric disorders: results from the national epidemiologic survey on alcohol and related conditions. J. Clin. Psychiatry 66, 564-574. doi: 10.4088/JCP.v66n0504

Potenza, M. N. (2013). Biological contributions to addictions in adolescents and adults: prevention, treatment, and policy implications. J. Adolesc. Health 52, S22-S32. doi: 10.1016/j.jadohealth.2012.05.007

Raylu, N., and Oei, T. P. (2002). Pathological gambling: a comprehensive review. Clin. Psychol. Rev. 22, 1009-1061. doi: 10.1016/S0272-7358(02)00101-0

Raylu, N., Oei, T. P., Loo, J. M., and Tsai, J. S. (2016). Testing the validity of a cognitive behavioral model for gambling behavior. J. Gambl. Stud. 32, 773-788. doi: 10.1007/s10899-015-9567-5

Ryan, T. P. (1997). Modern Regression Methods. New York, NY: Wiley.

Scholes-Balog, K. E., Hemphill, S. A., Dowling, N. A., and Toumbourou, J. W. (2014). A prospective study of adolescent risk and protective factors for problem gambling among young adults. J. Adolesc. 37, 215-224. doi: 10.1016/j.adolescence.2013.12.006

Sharma, L., Kohl, K., Morgan, T. A., and Clark, L. A. (2013). Impulsivity: relations between self-report and behavior. J. Pers. Soc. Psychol. 104, 559-575. doi: $10.1037 / \mathrm{a} 0031181$

Shead, N. W., Derevensky, J. L., and Gupta, R. (2010). Risk and protective factors associated with youth problem gambling. Int. J. Adolesc. Med. Health 22, 39-58.

Shenassa, E. D., Paradis, A. D., Dolan, S. L., Wilhelm, C. S., and Buka, S. L. (2012). Childhood impulsive behavior and problem gambling by adulthood: a 30-year prospective community-based study. Addiction 107, 160-168. doi: 10.1111/j.1360-0443.2011.03571.x

Slutske, W. S., Moffitt, T. E., Poulton, R., and Caspi, A. (2012). Undercontrolled temperament at age 3 predicts disordered gambling at age 32: a longitudinal study of a complete birth cohort. Psychol. Sci. 23, 510-516. doi: $10.1177 / 0956797611429708$

Steinberg, L., Graham, S., O’Brien, L., Woolard, J., Cauffman, E., and Banich, M. (2009). Age differences in future orientation and delay discounting. Child Dev. 80, 28-44. doi: 10.1111/j.1467-8624.2008.01244.x

Ste-Marie, C., Gupta, R., and Derevensky, J. L. (2006). Anxiety and social stress related to adolescent gambling behavior and substance use. J. Child Adolesc. Subst. Abuse, 15, 55-74. doi: 10.1300/J029v15n04_03

Stewart, S. H., Zack, M., Collins, P., Klein, R. M., and Fragopoulos, F. (2008). Subtyping pathological gamblers on the basis of affective motivations for gambling: relations to gambling problems, drinking problems, and affective motivations for drinking. Psychol. Addict. Behav. 22, 257-268. doi: 10.1037/0893-164X.22.2.257

Stinchfield, R. (2010). A critical review of adolescent problem gambling assessment instruments. Int. J. Adolesc. Med. Health, 22, 77-93. 
Tabachnick, B. G., and Fidell, L. S. (2013). Using Multivariate Statistics, 6th Edn. Boston, MA: Pearson.

Takamatsu, S. K., Martens, M. P., and Arterberry, B. J. (2016). Depressive symptoms and gambling behavior: mediating role of coping motivation and gambling refusal self-efficacy. J. Gambl. Stud. 32, 535-546. doi: $10.1007 /$ s10899-015-9562-x

Toneatto, T., and Pillai, S. (2016). Mood and anxiety disorders are the most prevalent psychiatric disorders among pathological and recovered gamblers. Int. J. Ment. Health Addict. 14, 217-227. doi: 10.1007/s11469-016-9647-5

Toplak, M. E., Liu, E., MacPherson, R., Toneatto, T., and Stanovich, K. E. (2007). The reasoning skills and thinking dispositions of problem gamblers: a dualprocess taxonomy. J. Behav. Decis. Mak. 20, 103-124. doi: 10.1002/bdm.544

van den Bos, R., Davies, W., Dellu-Hagedorn, F., Goudriaan, A. E., Granon, S., Hombergi, J., et al. (2013). Cross-species approaches to pathological gambling: a review targeting sex differences, adolescent vulnerability and ecological validity of research tools. Neurosci. Biobehav. R. 37, 2454-2471. doi: 10.1016/j.neubiorev.2013.07.005

Volberg, R. A. (1993). Gambling and Problem Gambling Among Adolescents In Washington State. Report to the Washington State Lottery.

Volberg, R., A., Gupta, R., Griffiths, M. D., Olason, D. T., and Delfabbro, P. (2010). An international perspective on youth gambling prevalence studies. Int. J. Adolesc. Med. Health 22, 3-38.

Welte, J. W., Barnes, G. M., Tidwell, M. C., Hoffman, J. H., and Wieczorek, W. F. (2015). Gambling and problem gambling in the United States: changes between 1999 and 2013. J. Gambl. Stud. 31, 695-715. doi: 10.1007/s10899-014-9471-4

Whiteside, S. P., Lynam, D. R., Miller, J. D., and Reynolds, S. K. (2005). Validation of the UPPS impulsive behavior scale: a four-factor model of impulsivity. Eur. J. Pers. 19, 559-574. doi: 10.1002/per.556
Wiehler, A., and Peters, J. (2015). Reward-based decision making in pathological gambling: the roles of risk and delay. Neurosci. Res. 90, 3-14. doi: 10.1016/j.neures.2014.09.008

Williams, A. D., Grisham, J. R., Erskine, A., and Cassedy, E. (2012). Deficits in emotion regulation associated with pathologic al gambling. Br. J. Clin. Psychol. 51, 223-238. doi: 10.1111/j.2044-8260.2011.02022.x

Winters, K. C., Stinchfield, R. D., and Fulkerson, J. (1993). Toward the development of an adolescent gambling problem severity scale. J. Gambl. Stud. 9, 63-84. doi: 10.1007/BF01019925

Winters, K. C., Stinchfield, R. D., and Kim, L. G. (1995). Monitoring adolescent gambling in Minnesota. J. Gambl. Stud. 11, 165-183. doi: 10.1007/BF02107113

Wood, R. T., and Griffiths, M. D. (2007). A qualitative investigation of problem gambling as an escape-based coping strategy. Psychol. Psychother. 80, 107-125. doi: 10.1348/147608306X107881

Wulfert, E., Roland, B. D., Hartley, J., Wang, N., and Franco, C. (2005). Heart rate arousal and excitement in gambling: Winners versus losers. Psychol. Addict. Behav. 19, 311-316. doi: 10.1037/0893-164X.19.3.311

Conflict of Interest Statement: The authors declare that the research was conducted in the absence of any commercial or financial relationships that could be construed as a potential conflict of interest.

Copyright (c) 2017 Nigro, Cosenza and Ciccarelli. This is an open-access article distributed under the terms of the Creative Commons Attribution License (CC BY). The use, distribution or reproduction in other forums is permitted, provided the original author(s) or licensor are credited and that the original publication in this journal is cited, in accordance with accepted academic practice. No use, distribution or reproduction is permitted which does not comply with these terms. 